\title{
Un nouveau Microparasellidae (Crustacé Isopode) du Haut-Atlas; aspects phylogénétiques et biogéographiques
}

\author{
M. Yacoubi-Khebiza', M. Boulanouar ${ }^{1} \&$ N. Coineau ${ }^{2}$ \\ 'Université de Marrakech, Laboratoire d'Hydrobiologie et Ecologie, Faculté des Sciences Semlalia, B.P. \\ S 15, Marrakech, Maroc; ${ }^{2}$ Université P. et M. Curie, U.R.A. C.N.R.S. 21.26, Observatoire Océanologique \\ de Banyuls, Laboratoire Arago, 66650 Banyuls-sur-Mer, France
}

Keywords: Taxonomy, biogeography, Crustacea, interstitial Isopoda, Microcharon, High Atlas, Morocco

\begin{abstract}
The interstitial stygobiotic isopods of the genus Microcharon (Crustacea) are diversified in Morocco. A new species, $M$. ourikensis, is described. The new species shows some original characters for this genus: large size, strong elongation in the first male pleopod exopodal inner lobe, 5-dentate mandibular pars incisiva, and 5 spines on the last mandibular palp segment. The species is a member of the Ibero-Maghrebin messoulii group. It is an endemic representative of the Ourika valley groundwater in the Moroccan High Atlas. It derives from a littoral marine ancestor which could have colonized the continental subterranean water during Turonian or Senonian Tethyan regressions.
\end{abstract}

\section{Résumé}

Les Isopodes interstitiels stygobies du genre Microcharon (Crustacés Microparasellidae) sont diversifiés au Maroc. $M$. ourikensis, nouvelle espèce, est décrite. Elle présente des caractères originaux pour le genre tels que la grande taille, le fort allongement du lobe interne des pléopodes 1 du mâle dont l'exopodite est long, 5 dents à la pars incisiva mandibulaire et 5 épines au dernier article du palpe. L'espèce appartient au groupe hispano-maghrébin messoulii. Elle est endémique de la nappe souterraine de la vallée de l'Ourika, Haut-Atlas marocain. Elle dérive d'un ancêtre marin littoral qui aurait colonisé les eaux souterraines continentales lors des régressions marines du Turonien ou du Sénonien.

\section{Introduction}

Les Isopodes Microparasellidae stygobies interstitiels du genre Microcharon sont diversifiés au Maroc. L'espèce littorale $M$. marinus a tout d'abord été signalée des rivages méditerranéens
(Coineau, 1971; 1982; 1986). Puis Pesce et al. (1981) récoltent une espèce dans l'ouest du pays, près de Casablanca, tandis que Messouli (1984) rapporte la présence du genre dans les sources du Haouz. Une espèce inédite du genre est ensuite recueillie dans les eaux souterraines des puits de Marrakech (Boulanouar, 1986; Boutin \& Boulanouar, 1984; Boutin, 1993a).

De même, au cours de prospections menées dans le cadre d'une étude écologique et biogéographique de la stygofaune interstitielle du HautAtlas de Marrakech, puis de recherches récentes et actuellement en cours, Yacoubi-Khebiza (1990) trouve plusieurs espèces inédites dans les vallées du Zat, de l'Ourika, du N'Fis et d'Amizmiz et montre qu'une espèce différente caractérise chacune de ces vallées, en plus d'une espèce récemment décrite, commune à quelques vallées et aux Jbilet (Boulanouar et al., 1995). L'une de ces espèces, provenant de la nappe souterraine de l'Oued Ourika, est décrite ci-dessous. Puis nous envisageons ses affinités et son origine.

Microcharon ourikensis n. sp. (Figs. 1-3)

Matériel. - La description se base sur l'observation et la dissection de 3 mâles et 6 femelles; des jeunes et des individus des deux sexes ont été en outre récoltés, dans plusieurs puits le long de la vallée de l'Ourika, à une altitude comprise entre 900 et $1200 \mathrm{~m}$ (Yacoubi rec., 1993-94). La série syntype reste provisoirement dans la collection des auteurs pour les besoins d'études phylogénétiques. Ultérieurement, une partie de cette série sera déposée dans les collections nationales de Rabat et de Paris. 
Derivatio nominis. - Le nom de cette espèce se rapporte à celui de l'Oued Ourika.

Description. - Cette espèce se remarque immédiatement par sa forte taille allant de 2 à $2,2 \mathrm{~mm}$ pour les mâles, et de 2,1 à $2,6 \mathrm{~mm}$ pour les femelles.

Antennes 1 (Fig. 1E): Elles se composent de 6 articles, le premier étant élargi et portant 2 soies courtes dont une pennée; le second, long et plus étroit, ne présente que 2 petites soies pennées et 2 minuscules soies lisses subdistales; la longe tige pennée terminale insérée sur un socle en relief dépasse le niveau médian de l'article 5; article 3 glabre, le suivant muni de 2 soies latérales et d'une courte soie plumeuse; l'aesthétasque inséré sur l'article 5 est relativement court, contrairement à la lame distale du 6e segment qui porte un bouquet de 3 soies et une soie pennée subterminale. Aucun dimorphisme sexuel n'est à noter chez cette espèce en ce qui concerne le nombre d'aesthétasques.

Antennes 2: Tous les individus sont amputés du fouet. Le $3 \mathrm{e}$ article du pédoncule montre un exopodite relativement court.

Mandibules (Fig. 1C-D): La pars incisiva est armée de 5 dents, donnant l'impression que la dent surnuméraire provient de la subdivision de la dent la plus distale; la lacinia mobilis de la mandibule gauche se termine par les 4 dents usuelles; la mandibule gauche présente 2 tiges épaisses festonnées, 3 soies lisses et 2 longues soies barbelées entre le complexe incisif et le processus molaire, tandis que la droite est armée de 3 tiges festonnées, 2 soies nues et 2 soies barbelées. Le processus molaire conique se termine par les 3 soies barbelées normales. Le palpe possède 2 épines barbelées à l'article 2; l'article 3 donne naissance à 4 épines à gauche et 5 épines à droite, de longueur décroissante vers l'extrémité, et à une petite rangée de cils à la mandibule droite seulement.

Maxilles 1 (Fig. 1B): L'endite interne, étroit, se termine par 4 soies lisses; la marge interne offre une série de 4 soies dans la région subdistale, tandis que la marge externe en montre 4 . L'endite externe présente une série de 11 épines terminales dont 4 lisses et 1 ciliée en son extrémité du côté interne, 1 épine médiane arrondie à l'extrémité et munie de cils terminaux et 4 épines nues un peu plus courtes plus 1 épine barbelée côté externe; 4 fines soies subdistales au bord interne; 7 fines soies réparties le long du bord externe.

Maxilles 2 (Fig. 1A): L'endite interne, un peu plus long et plus large que les endites médian et externe, porte 5 soies apicales lisses et 6 soies latérales; les 2 autres endites se terminent par 4 soies arquées et pectinées à leur extrémité, dont 2 sont très longues.

Maxillipèdes (Fig. 1F). Le sommet de l'épipodite atteint le milieu du $2 \mathrm{e}$ article du palpe. Endite court se terminant au-dessous de la limite des $2 \mathrm{e}$ et $3 \mathrm{e}$ segments du palpe; il est muni de 7 épines distales et son repli est garni de nombreuses soies fines, en plus des 2 rétinacles. Le palpe compte 5 articles: article basal très court et très large, trapu, avec 1 soie de chaque côté; le second s'élargit progressivement de la base au sommet et montre 2 soies subterminales; le $3 \mathrm{e}$, au contraire, voit sa largeur diminuer de la base vers la zone distale et porte les 3 soies internes et la soie distale externe; les 2 derniers articles étroits offrent la chétotaxie usuelle; le $4 \mathrm{e}$ article est relativement long.

Péréiopodes (Fig. 2A-B): Ils ont été le plus souvent éliminés lors des prélèvements. Ils sont relativement longs et grêles comme chez la plupart des espèces d'eau douce. Le basis est peu élargi et possède de 1 à 4 soies dont 2 pennées sur la crête tergale. La chétotaxie des différents articles est conforme au canevas habituel chez le genre; les 2 griffes terminales sont longues et très inégales.

Pléotelson (Fig. 3C): Marges latérales subrectilignes, à peine renflées au niveau du $1 / 3$ proximal; marge distale décrivant un triangle à angle principal obtus, mais cet angle devient un peu plus aigu et dépasse en une pointe obtuse dans la zone médiane; les 2 soies pennées dorsales subterminales sont présentes.

Pléopodes 1 du mâle (Fig. 3A): Ils sont très longs. La région basale coalescente est élargie; les exopodites sont séparés et individualisés sur une longueur supérieure au 1/3 de la longueur totale de l'appendice. Le repli (Cvetkov, 1968) se situe loin de l'extrémité et se termine loin de 

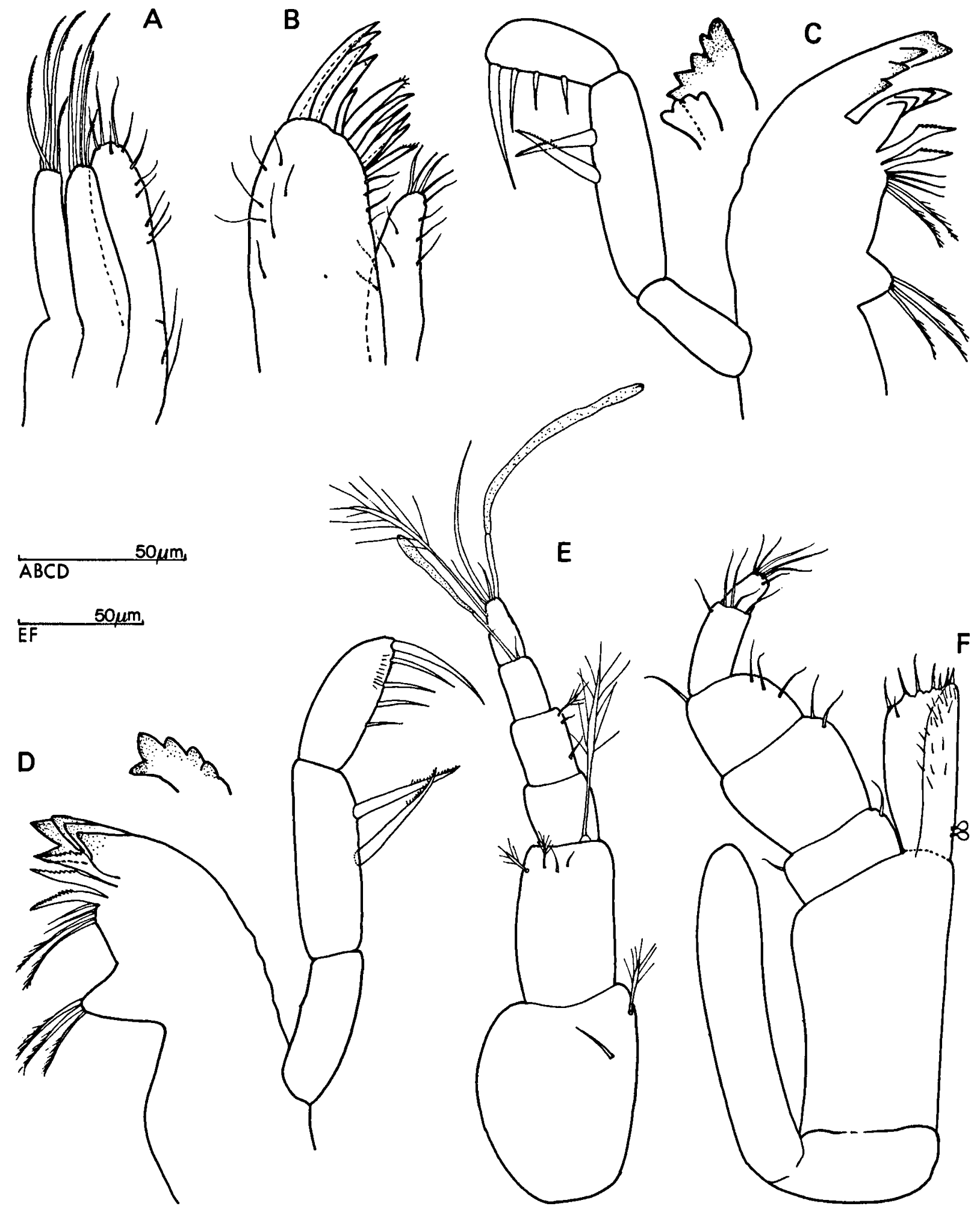

Fig. 1. Microcharon ourikensis n. sp.: A, maxille $2 \sigma ; \mathrm{B}$, maxille $1 \%$; , mandibule gauche et détail de la pars incisiva et de la lacinia mooiis $\sigma^{\prime} ; \mathrm{D}$, mandibule droite et vue ventrale de la pars incisiva; $\mathrm{E}$, antenne $1 \sigma^{\circ} ; \mathrm{F}$, maxillipède $\sigma^{\prime}$. 


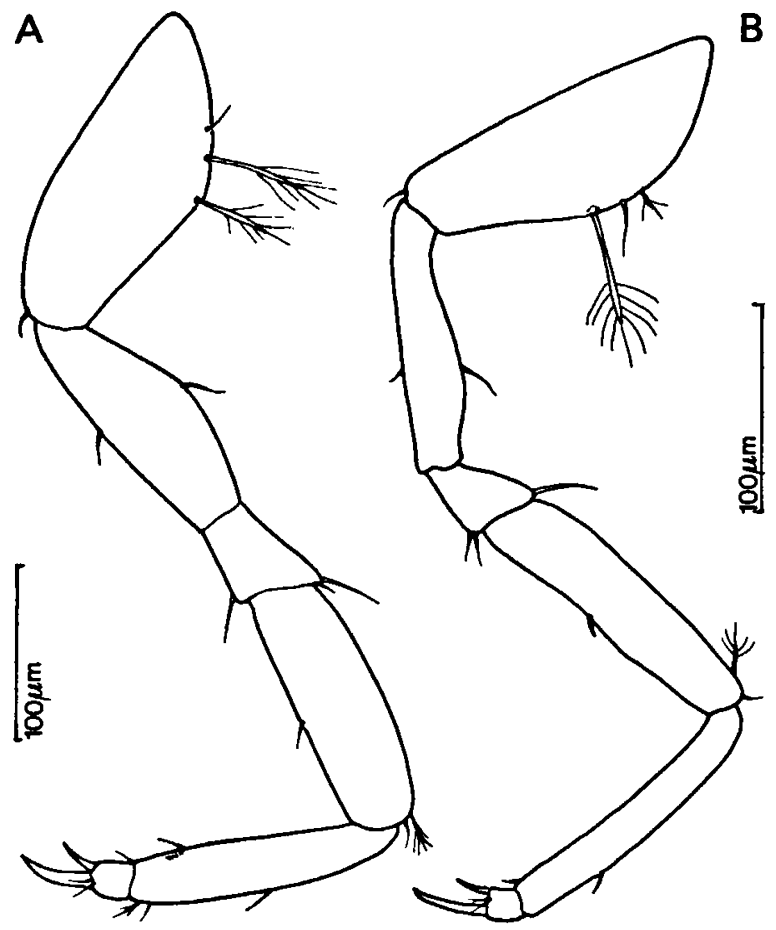

Fig. 2. Microcharon ourikensis n. sp.: A, péréiopode 3 \&; B, péréiopode 5 \%.

celle-ci; le lobe interne est très allongé et se termine en une région conique portant 2 soies distales peu différentes en longueur et relativement courtes; les 3 soies habituellement localisées sur le repli lui-même, se situent toutes en dehors de ce repli; les 2 soies 3 et 4 de Cvetkov se répartissent dans la zone médiane du cône, donc à distance de l'extrémité.

Pléopodes 2 du mâle (Fig. 3D): Le sympode se termine par un lobe arrondi prononcé. L'apex de l'appendix masculina, modérément effilé, atteint la marge distale de ce lobe, sans le dépasser.

Pléopode 2 de la femelle (Fig. 3B): nettement plus long que large; marge distale convexe, bilobée, présentant deux soies peu développées.

Pléopodes 3 (Fig. 3E): Endopodite renflé, glabre. Exopodite à 2 articles, le dernier à peine arqué, dont la soie terminale dépasse de peu l'endopodite.

Tous les exemplaires recueillis ont perdu leurs uropodes, qui tombent très facilement chez ce genre, au cours des prélèvements. En dehors des aesthétasques des antennes 1 , aucun dimorphisme sexuel n'á été décelé sur les autres appendices bucaux ou thoraciques.

\section{Discussion}

Microcharon ourikensis n. sp. se distingue notablement de l'espèce marocaine $M$. boutini d'une part, ainsi que de la plupart des autres espèces connues actuellement. Elle se rapproche, par contre, de $M$. karamani d'Algérie (Pesce \& Tetè, 1978) et de $M$. hispanicus d'Espagne (Pesce \& Galassi, 1988), en particulier par les caractéristiques des pléopodes 1 du mâle dont le lobe interne long prend la forme d'un cône, plus long chez l'espèce marocaine. Les trois espèces présentent en effet un repli éloigné de l'extrémité de l'exopodite, ainsi qu'un lobe distale fortement allongé. Cet allongement est moindre chez $M$. hispanicus et $M$. karamani, de telle sorte que les 3 soies distales occupent une position terminale (soies 1, 2 et 3 de Cvetkov, 1968), alors que 2 soies seulement (soies 1,2 ) sont terminales chez $M$. ourikensis, la $3 \mathrm{e}\left(\mathrm{n}^{\circ} 3\right)$ se situant nettement en retrait de la marge. La soie 4, selon Cvetkov (1968), au lieu d'être latérale comme chez la plupart des espèces, devient submédiane, reste distale pour les espèces algérienne et espagnole citées, et se trouve à mi-distance entre les extrémités de l'exopodite et du repli, c'est-à-dire fort loin de l'extrémité pour M. ourikensis. Quant aux soies situées habituellement dans la zone médiane ou proximale du repli, 2 restent à ce niveau chez $M$. hispanicus, l'autre se situant hors du repli, 2 sont issues de la région distale du repli, la 3e étant à sa limite chez $M$. karamani, tandis que chez $M$. ourikensis, les 3 soies se localisent en dehors du repli, au niveau de sa région distale. Cette disposition résulte de la position du repli très éloigné de l'apex de l'exopodite et du fort allongement du lobe interne qui est maximum chez $M$. ourikensis. $M$. ourikensis se distingue en outre de $M$. hispanicus par la forme du lobe interne plus arrondi et moins allongé chez cette dernière espèce.

$M$. ourikensis se différencie de $M$. karamani par: sa taille plus élevée (il s'agit de l'une ou de la plus longue espèce connue), la tige pennée 


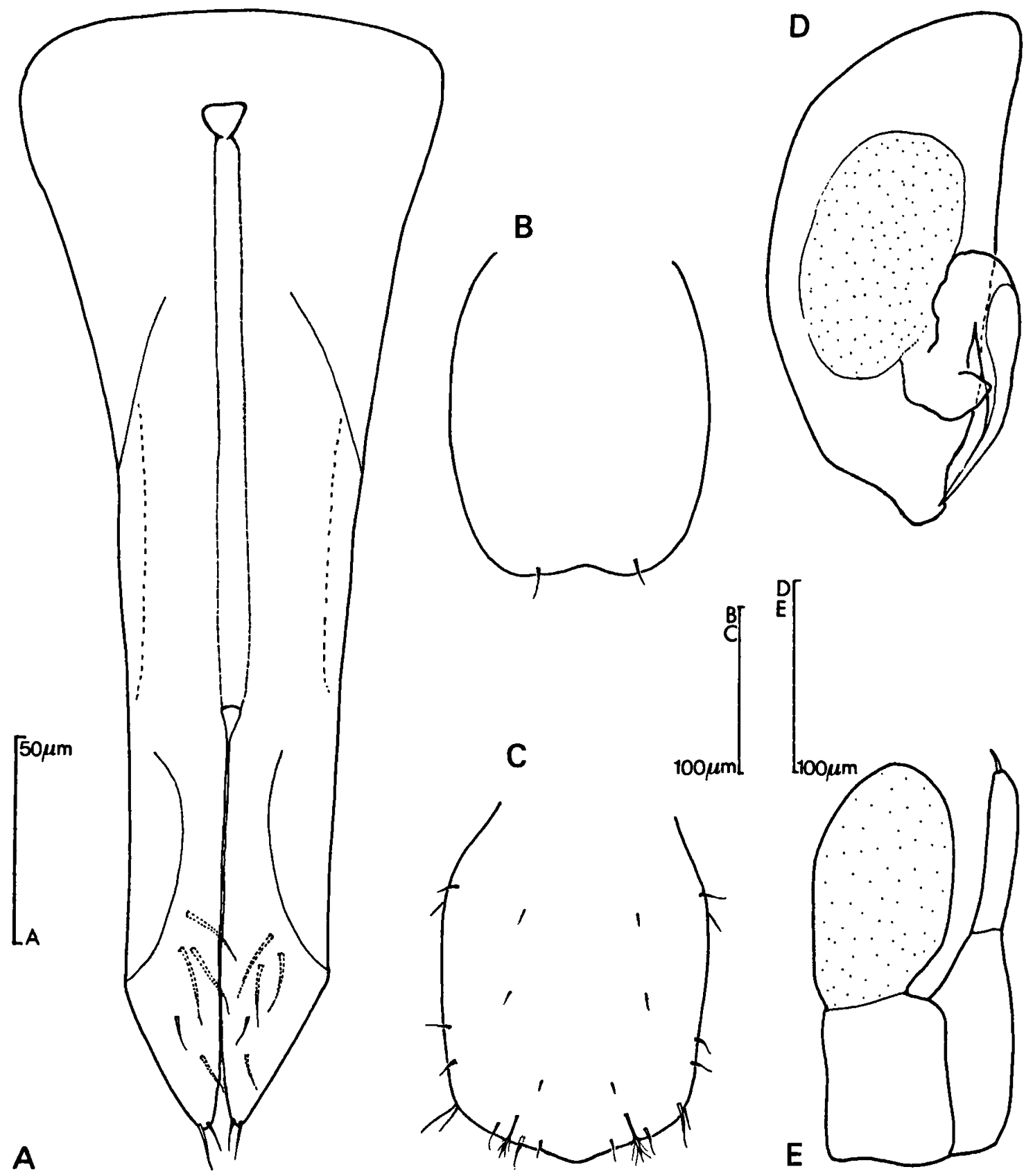

Fig. 3. Microcharon ourikensis n. sp.: A, pléopode 1 ơ; B, pléopode 2 \%; C, pléotelson ơ; D, pléopode 2 ơ; E, pléopode 3 \&.

pédonculée de l'antenne 1 (sur le $2 \mathrm{e}$ article) un peu plus courte, la pars incisiva mandibulaire composée de 5 dents, l'armature de 5 épines du 3e article du palpe de la mandibule droite, l'absence de la soie pectinée terminale de l'endite interne de la maxille 2 qui ne compte que 5 soies apicales (au lieu de 6), la forme du pléotelson au contour terminal subtriangulaire (légèrement arrondi pour M. karamani), la grande longueur de l'exopodite du pléopode 1 du mâle, sans doute en 
relation avec l'allongement du lobe interne et la position non terminale du repli, le pléopode $2 \mathrm{du}$ mâle dont l'apex de l'appendix masculina atteint l'extrémité du lobe du sympode, et le pléopode 2 de la femelle plus allongé.

\section{Remarques phylogénétiques et biogéographiques}

Microcharon ourikensis appartient au groupe d'espèces hispano-maghrébines qui se caractérisent par une synapomorphie remarquable concernant le fort allongement du lobe interne des pléopodes 1 du mâle. Ces espèces sont cependant considérées comme porteuses des caractères les plus plésiomorphes du genre Microcharon (Coineau, 1994; Boulanouar et al., 1995; Coineau et al., en préparation).

La nouvelle espèce est endémique de la vallée de l'Oued Ourika; elle se répartit le long de ce cours d'eau seulement; ce sont d'autres espèces qui habitent dans le cours souterrain des autres oueds du Haut-Atlas de Marrakech (Yacoubi Khebiza, 1990; Boulanouar et al., 1995).

Les espèces du genre qui occupent les nappes souterraines d'eau douce ont eu des ancêtres marins interstitiels littoraux. Le modèle biphase de colonisation et d'évolution constitue l'un des modèles susceptibles d'expliquer leur origine et leur évolution (Coineau, 1985; Boutin \& Coineau, 1990; Notenboom, 1991; Coineau \& Boutin, 1992; Coineau, 1994). Ce modèle montre que l'établissement des espèces interstitielles stygobies dans les eaux douces souterraines à partir d'ancêtres marins s'est accompli en deux temps. Durant la première phase du scénario, qui correspond à une dispersion active, un ancêtre marin de surface colonise le fond sableux de la zone littorale marine et devient interstitiel. Au cours de la seconde phase, une partie des populations de cet ancêtre interstitiel littoral peut rester sur place et devenir progressivement et passivement dulçaquicole pendant une régression marine de la Téthys. Des processus vicariants interviennent au terme de la régression, dès que le flux génique est interrompu entre le nouveau stygobionte d'eau douce souterraine et le reste de la population demeurée littorale. Cette dernière phase, liée à une régression marine, correspond au "Regression Model Evolution" (Stock, 1977; 1980; Botosaneanu \& Holsinger, 1991).

Ce scénario biogéographique est d'autant plus plausible, dans le cas de Microcharon, qu'il existe dans la nature actuelle plusieurs espèces marines de fonds sableux peu profonds, peu éloignés des côtes, ainsi que de nombreuses espèces littorales vivant dans les sables des plages du rivage marin (Coineau, 1986; 1992; 1994). En outre, quelques études biogéographiques ont déjà montré que les espèces aux caractères les plus dérivés proviennent des régressions marines les plus récentes: Tortonien, Pliocène en France et en Italie (Dole \& Coineau, 1987; Coineau, 1992). Au contraire, les espèces montrant de nombreux caractères primitifs sont liées à des retraits de la Téthys plus précoces, datant de l'Eocène, voire du Turonien ou du Sénonien (Coineau, 1994). Des résultats similaires ont été mis en évidence pour divers groupes de Crustacés interstitiels tels que le genre Pseudoniphargus en Espagne et au Maroc (Stock, 1980; Boutin \& Coineau, 1988; Notenboom, 1988; 1991), les espèces de la famille des Amphipodes Metacrangonyctidae (Boutin \& Messouli, 1988a; 1988b; Boutin, 1994; Boutin et al., 1992; Boutin, 1993a; Messouli, 1994), des espèces des familles d'Amphipodes Artesidae, Hadziidae, Sebidae (Holsinger, 1986; 1992; 1994), ou encore les Parabathynellidae (Camacho \& Coineau, 1989), et les Cirolanidae du Bassin Méditerranéen (Boutin, 1993b).

Tous ces résultats s'appuient sur des recherches multidisciplinaires: établissement de la monophylie du groupe étudié et des cladogrammes, recherches d'événements géologiques ou paléogéographiques susceptibles d'être intervenus en tant que barrières responsables des divergences évolutives, et construction de scénarios biogéographiques expliquant l'origine et l'histoire évolutive des espèces étudiées.

Comme les autres espèces du groupe messoulli auquel elle appartient, $M$. ourikensis proviendrait d'un ancêtre marin qui vivait très probablement dans les eaux interstitielles des sables littoraux de la Téthys au Turonien ou au Sénonien; de larges aires du Maroc ont été submergées lors de ces 
deux époques et de vastes golfes ont pénétré notamment à l'emplacement actuel de l'Atlas, c'est-à-dire dans la zone habitée aujourd'hui par M. ourikensis (Choubert \& Faure-Muret, 1962; Michard, 1976; Coineau, 1994). Les populations ancestrales de cette espèce ont dû pénétrer dans les eaux douces souterraines lors de l'une des régressions turonienne ou sénonienne (Michard, 1976; Choubert \& Faure-Muret, 1962). Ultérieurement, l'orogenèse atlasique a pu entraîner des phénomènes de vicariance qui ont été à l'origine de $M$. ourikensis.

\section{Conclusion}

L'Isopode interstitiel Microcharon ourikensis apparaît comme une espèce très originale au sein du genre, notamment par les caractéristiques de ses premiers pléopodes qui ont conservé des caractères plésiomorphes chez le mâle, mais qui se distinguent par une apomorphie remarquable portant sur le lobe interne. Elle montre des affinités avec $M$. hispanicus, du sud de l'Espagne, et $M$. karamani, qui provient d'Algérie. M. ourikensis est endémique de la nappe alluviale de l'Oued Ouri$\mathrm{ka}$, en moyenne altitude, dans le Haut-Atlas de Marrakech. La colonisation des eaux douces par les populations marines ancestrales de cette espèce pourrait remontuer au Sénonien ou au Turonien.

\section{Remerciements}

Ces travaux se poursuivent dans le cadre de l'Action intégrée Franco-Marocaine $n^{\circ}$ 93/643, du GDR 88 "Evolution des organismes microscopiques" et du Contrat ACC-SV n 7 "Diversité fonctionelle et évolution en milieu souterrain".

\section{Bibliographie}

Boulanouar, M., 1986. Etude écologique comparée de quelques puits de la région de Marrakech. Impact des pollutions sur la zoocénose des puits: 1-159 (thèse $3 \mathrm{e}$ cycle, Univ. Marrakech).

Boulanouar, M., M. Yacoubi, M. Messouli \& N. Coineau, 1995. Un nouveau Microcharon (Isopoda, Janiroidea) du Maroc. Origine et biogéographie historique. Contr. Zool., 65(1): 53-64.

Botosaneanu, L. \& R. Holsinger, 1991. Some aspects con- cerning the colonization of the subterranean realm - especially of subterranean waters: a response to Rouch \& Danielopol, 1987. Stygologia, 6(1): 11-39.

Boutin, C., 1993a. Biogéographie historique des Crustacés Malacostracés stygobies du Maroc: 1-262 (thèse Doct., Univ. Lyon I).

Boutin, C., 1993b. Biogéographie historique des Crustacés Isopodes Cirolanidae stygobies du groupe Typhlocirolana dans le bassin méditerranéen. C. r. hebd. Séanc. Acad. Sci. Paris, Sci. Vie, 316(12): 1505-1510.

Boutin, C., 1994. Biogeography of the Metacrangonyctid amphipods in North Africa. Hydrobiologia, 287(1): 4964.

Boutin, C. \& M. Boulanouar, 1984. Premières données sur la faune des puits de Marrakech. Verh. int. Verein Limnol., 22: $1762-1765$.

Boutin, C. \& N. Coineau, 1988. Pseudoniphargus maroccanus n. sp. (subterranean amphipod), the first representative of the genus in Morocco. Phylogenetic relationships and palaeobiogeography. Crustaceana, Suppl. 13: 1-19.

Boutin, C. \& N. Coineau, 1990. "Regression Model", "Modèle biphase" d'évolution et origine des micro-organismes stygobies interstitiels continentaux. Revue Micropaléont., 33(3/4): 303-322.

Boutin, C. \& M. Messouli, 1988a. Longipodacrangonyx maroccanus n. gen. n. sp., nouveau représentant du groupe Metacrangonyx dans les eaux souterraines du Maroc. Crustaceana, Suppl. 13: 256-271.

Boutin, C. \& M. Messouli, 1988b. Metacrangonyx gineti n. sp. d'une source du Haut-Atlas marocain et la famille des Metacrangonyctidae n. fam. (Crustacés Amphipodes stygobies). Vie Milieu, 38(1): 67-84.

Boutin, C., M. Messouli \& N. Coineau, 1992. Phylogénie et biogéographie évolutive d'un groupe de Metacrangonyctidae, Crustacés Amphipodes stygobies du Maroc. II. Cladistique et paléobiogéographie. Avec l'examen comparatif de plusieurs logiciels de parcimonie. Stygologia, 7(3): 159-177.

Camacho, A.I. \& N. Coineau, 1989. Les Parabathynellacés (Crustacés Syncarides) de la Péninsule Ibérique. Répartition et paléobiogéographie. Mém. Biospéol., 16: 111124.

Choubert, G. \& A. Faure-Muret, 1962. Evolution du domaine atlasique marocain depuis les temps paléozoïques. In: Livre à la mémoire du Professeur Paul Fallot, 1: 447-527 (Mém. hors série, Soc. géol. France, Paris).

Coineau, N., 1971. Les Isopodes interstitiels. Documents sur leur écologie et leur biologie. Mém. Mus. natn. Hist. nat., (N.S., A) 64: 1-170.

Coineau, N., 1982. Microcharon Kar. et Angeliera Chap. et Delam., Crustacés Isopodes Asellotes interstitiels Microparasellides. Documents pour un atlas zoogéographique du Languedoc-Roussillon, 24: 1-4.

Coineau, N., 1985. Colonisation du milieu souterrain continental par les organismes d'origine marine. Moyen d'accès par le milieu interstitiel. Bull. Soc. Biospéol., 8: 26-30. 
Coineau, N., 1986. Isopoda: Asellota: Janiroidea. In: L. Botosaneanu (ed.), Stygofauna mundi: 465-472 (E.J. Brill/W. Backhuys, Leiden).

Coineau, N., 1992. Biogéographie évolutive du Crustacé interstitiel Microcharon (Isopoda, Janiroidea) dans l'ouest du Bassin Méditerranéen. Bull. Inst. océanogr., Monaco, 9: 101-114.

Coineau, N., 1994. Evolutionary biogeography of the microparasellid isopod Microcharon (Crustacea) in the Mediterranean Basin. In: Biogeography of subterranean crustaceans: the effects of different scales. Summer Meeting of the Crustacean Society, Charleston, June 1992. Hydrobiologia, 287(1): 79-96.

Coineau, N. \& C. Boutin, 1992. Biological processes in space and time. Colonization, evolution and speciation in interstitial stygobionts. In: A.I. Camacho (ed.), The natural history of biospeleology. Mus. nac. Cienc. nat., CSIC Ed., Madrid, Monografias, 7: 423-451.

Coineau, N., M. Boulanouar, M. Yacoubi \& C. Boutin (in prep.). Evolution and historical biogeography of the interstitial isopod Microcharon (Crustacea) in Morocco.

Cvetkov, L., 1968. Morphologie des premiers pléopodes, évolution et position systématique du genre Microcharon (Crustacea, Isopoda). Izv. zool. Inst. Sof., 27: 107-140.

Dole, M.-J. \& N. Coineau, 1987. L'Isopode Microcharon (Crustacea, Isopoda) abondant dans les eaux interstitielles de l'est Lyonnais. M. reginae n. sp., écologie et biogéographie. Stygologia, 3(3): 200-216.

Holsinger, J.R., 1986. Zoogeographic patterns of North American subterranean crustaceans. In: R.D. Gore \& K.L. Heck (eds.), Crustacean Issues 4, Crustacean biogeography: 85-106 (A.A. Balkema, Rotterdam).

Holsinger, J.R., 1992. Four new species of subterranean amphipod crustaceans (Artesidae, Hadziidae, Sebidae) from Texas, with comments on their phylogenetic and biogeographic relationships. Texas mem. Mus., Speleol. Monogr., 3: 1-22.

Holsinger, J.R., 1994. Pattern and process in the biogeography of subterranean amphipods. In: Biogeography of subterranean crustaceans: the effects of different scales. Summer Meeting of the Crustacean Society, Charleston,
June 1992. Hydrobiologia, 287(1): 131-145.

Messouli, M., 1984. Recherches de la faune stygobie des sources de la région de Marrakech. Mém. DEA, Univ. Marrakech: 1-35.

Messouli, M., 1994. Evolution, phylogénie et biogéographie historique des Metacrangonyctidae, Crustacés Amphipodes stygobies du Nord de l'Afrique et des régions voisines: 1-309 (thèse Doct. d'Etat, Univ. Marrakech).

Michard, A., 1976. Eléments de géologie du Maroc. Notes Mém. Serv. géol. Maroc, 252: 1-408.

Notenboom, J., 1988. Phylogenetic relationships and biogeography of the groundwater-dwelling amphipod genus Pseudoniphargus (Crustacea), with emphasis on the Iberian species. Bijdr. Dierk., 58(2): 159-204.

Notenboom, J., 1991. Marine regressions and the evolution of groundwater dwelling amphipods (Crustacea). J. Biogeography, 18: 437-454.

Pesce, G.L. \& D.P. Galassi, 1988. Microparasellids of Spain (Crustacea Isopoda: Janiroidea). Stygologia, 4: 307-331.

Pesce, G.L. \& P. Tetè, 1978. Recherches en Afrique de l'Institut de Zoologie de l'Aquila (Italie). I. Microparasellides d'Algérie (Crustacea: Isopoda). Rev. Zool. afr., 92(4): 992-1002.

Pesce, G.L., P. Tetè \& M. de Simone, 1981. Ricerche faunistiche in acque sotterranee del Maghreb (Tunisia, Algeria, Marocco) e dell'Egitto. Natura, Milano, 72(1-2): 63-98.

Stock, J.H., 1977. The taxonomy and zoogeography of the hadziid Amphipoda, with emphasis on the West Indian taxa. Stud. Fauna Curaçao, 55: 1-130.

Stock, J.H., 1980. Regression model evolution as exemplified by the genus Pseudoniphargus (Amphipoda). Bijdr. Dierk., 50(1): 105-144.

Yacoubi Khebiza, M., 1990. Ecologie et biogéographie des biocénoses aquatiques des nappes alluviales de quelques vallées du Haut-Atlas de Marrakech (Maroc). Paléobiogéographie des Crustacés phréatobies: 1-246, annexes 1-9 (thèse 3e cycle, Univ. Marrakech).

Reçu le 9 juillet 1996 